\title{
The Influence of molecular design on structure-property relationships of a supramolecular polymer prodrug.
}

\author{
Kelsey G.DeFrates ${ }^{1, \dagger}$, Joakim Engström ${ }^{1,2, \dagger}$, Nivedina A. Sarma ${ }^{2}$, Athiyya Umar ${ }^{1}$, Jisoo Shin ${ }^{1,2}$, Jing \\ Cheng $^{1,2}$, Ahmad K. Omar ${ }^{2}$, Phillip B. Messersmith ${ }^{1,2,3^{*}}$ \\ ${ }^{1}$ Department of Bioengineering, University of California, Berkeley, Berkeley, CA 94720, USA. \\ ${ }^{2}$ Department of Materials Science and Engineering, University of California, Berkeley, Berkeley, CA \\ 94720, USA \\ ${ }^{3}$ Materials Sciences Division, Lawrence Berkeley National Laboratory, Berkeley, CA 94720, USA
}

${ }^{\dagger}$ Contributed equally as first authors to this work.

\author{
* Correspondence: University of California, Berkeley, 210 Hearst Mining Building Berkeley, CA 94720- \\ 1760, USA; E-mail: philm@berkeley.edu; Tel.: 510-643-9631
}

\begin{abstract}
Supramolecular self-assemblies of hydrophilic macromolecules functionalized with hydrophobic, structuredirecting components have long been used for drug delivery. In these systems, loading of poorly soluble compounds is typically achieved through physical encapsulation during or after formation of the supramolecular assembly, resulting in low encapsulation efficiencies and limited control over release kinetics that are predominately governed by diffusion and carrier degradation. To overcome these limitations, amphiphilic prodrugs that leverage a hydrophobic drug as both the therapeutic and structuredirecting component can be used to create supramolecular materials with higher loading and controlled release kinetics when biodegradable or enzymatically cleavable linkers are used. Here, we report the design, synthesis and characterization of a library of supramolecular polymer prodrugs based on poly(ethylene glycol) (PEG) and the pro-regenerative drug 1,4-dihydrophenonthrolin-4-one-3-carboxylic acid (DPCA). Structure-property relationships were elucidated through experimental characterization of prodrug behavior in both the wet- and dry-state, using scattering techniques and electron microscopy, and corroborated by coarse-grained modeling. Molecular architecture and hydrophobic-to-hydrophilic ratio of PEG-DPCA conjugates strongly influenced their physical state in water, ranging from fully soluble to supramolecular assemblies of micelles and nanofibers. Molecular design and supramolecular structure, in turn, were shown to dramatically alter hydrolytic and enzymatic release, bioactivity and cellular transport of DPCA. In addition to potentially expanding therapeutic options for DPCA through control of supramolecular assemblies, the resulting design principles elaborated here may inform the development of other supramolecular prodrugs based on hydrophobic small molecule compounds.
\end{abstract}




\section{Introduction}

In response to injury, most adult mammals are incapable of fully restoring normal tissue architecture and function. Instead, damage resulting from age-related degeneration, trauma, or disease is healed through a reparative process, that is marked by the production of non-specific fibrotic tissue ${ }^{1}$. While this new matrix may mitigate further damage and restore general barrier functions, its robust accumulation can greatly augment cell behavior and impair organ function. To mitigate fibrotic healing and achieve complete restoration of lost tissue, the field of tissue engineering has mainly focused on the use of biomaterials scaffolds, progenitor cells, and endogenous growth factors ${ }^{2-4}$. Despite success in small animal models and preclinical studies, translation of these strategies has been limited due to challenges associated with their industrial scale-up, high costs, cell sourcing, in vivo engraftment, and safety ${ }^{5}$. Recently, the use of smallmolecule drugs, capable of manipulating endogenous cells to facilitate regeneration rather than fibrotic repair, has emerged as a promising translational therapy for regenerative medicine ${ }^{6-11}$. However, potential biological targets capable of facilitating this response remain largely unknown and demands more fundamental understanding of both delivery systems and bioactivity.

Prior work from our group has demonstrated that the ubiquitously expressed transcription factor hypoxia inducible factor one-alpha (HIF-1 $\alpha$ ) is a potent, druggable target for mammalian tissue regeneration. It is well known that stabilization of this protein can lead to the expression of key genes associated with angiogenesis, cell differentiation, migration, metabolism, and tissue remodeling ${ }^{6-8}$. In most cell types, however, constitutive expression of HIF-1 $\alpha$ is counteracted by the action of prolyl hydroxylase domaincontaining enzymes (PHDs), which utilize molecular oxygen as a cofactor to hydroxylate the protein and trigger its degradation ${ }^{12,13}$. Recently, we demonstrated that transient stabilization of HIF-1a could be achieved via administration of the small-molecule PHD inhibitor 1,4-dihydrophenonthrolin-4-one-3carboxylic acid (DPCA $)^{6-8,14,15}$. Remarkably, adult mice with critical-sized ear wounds as well as significant periodontal bone loss exhibited complete regeneration of lost tissue without scar formation following subcutaneous administration of DPCA ${ }^{6-8}$. Murugan, et al. has also demonstrated that a multidrug cocktail containing DPCA can be used to regenerate amputated limbs in adult Xenopus laevis ${ }^{16}$. Since the normal wound healing cascade is highly conserved across organ systems, and DPCA's biological target HIF-1 $\alpha$ (via inhibition of PHD) is ubiquitously expressed across all cell types, we hypothesize that this drug is likely applicable for the treatment of a broad array of injuries and degenerative diseases.

Therefore, designing DPCA-based therapies that can be easily adapted to target diverse tissues and injuries is of great clinical need. The ability of hydrophobic drugs, such as DPCA, to partition into an organ of interest is dependent upon innate tissue characteristics such as $\mathrm{pH}$, vascular permeability, and perfusion rate, as well as the chemical properties of the drug such as lipid solubility, half-life, and protein-binding affinity ${ }^{17}$. While material vehicles have been developed to alter the pharmacokinetics of existing drugs, numerous studies have shown that the biodistribution of these nanocarriers is greatly affected by particle size, shape, and surface chemistry ${ }^{18,19}$. Modular drug delivery systems that allow these properties to be easily altered to allow for multi-tissue targeting, will have substantial utility in the field of regenerative medicine and bioengineering at large ${ }^{20}$. One such modular approach in drug delivery is to develop macromolecular prodrugs, created by covalently coupling a bioactive compound to a polymer, peptide, or polysaccharide..$^{21}$ In the case of small-molecule, hydrophobic drugs, this modification can lead to vast improvements in solubility and subsequent bioavailability ${ }^{21-23}$. Through careful molecular design, these prodrugs can also be driven to self-assemble into supramolecular aggregates due to their amphiphilic nature ${ }^{21,24}$. As drug delivery vehicles, these supramolecular structures offer several advantages over conventional soluble prodrugs, including decreased renal clearance and extended drug release kinetics. ${ }^{25,26}$ While many of these attributes may also be achieved through physical encapsulation of drug within nanocarriers such as polymeric micelles ${ }^{26}$, single-chain nanoparticles ${ }^{27}$, and surfactant micelles ${ }^{28}$, significantly higher drug loading, especially for poorly soluble compounds, is often achieved with prodrug aggregates, and any risk of carrier toxicity is eliminated. Recently, we reported on a self-assembling prodrug system using DPCA. In our initial design utilized DPCA as a therapeutic payload and structural element, by conjugating the hydrophobic drug to hydrophilic, linear poly(ethylene glycol) (PEG) chain. In aqueous 
solutions above a critical concentration, the PEG-DPCA conjugate assembled into well-defined nanofibers, consisting of a drug-rich core and polymer corona. ${ }^{6}$ Such non-spherical assemblies of prodrug structures have been rarely reported $12,24,29$, and when administered in vivo, we found that gradual DPCA release invoked multi-tissue regeneration of large ear wounds in adult mice ${ }^{6}$. However, we noted that this regeneration was highly dependent upon drug dosing and release kinetics, which were largely influenced by self-assembly behavior. With the current design, we have also found that we are limited to subcutaneous administration of viscous gels formed through entanglement and crosslinking of fibers. While this vehicle was adequate for treating ear wounds in our model study, we now seek to exploit the full therapeutic utility of DPCA to target clinically relevant injuries. Thus, this necessitates the development new delivery vehicles that can be used in alternative routes of administration such as intravenous, topical, and oral delivery. To create these carriers, we also seek to better understand the bioactivity and degradation behavior of existing DPCA prodrugs.

To meet this goal, this study evaluates the fundamental structure-property relationship between prodrug molecular architecture and clinical utility, to establish clear design principles for the development of future prodrug systems based on DPCA and related hydrophobic drugs. We present a detailed investigation into the assembly, stability, and bio-performance, of three PEG-DPCA conjugates, containing 1, 2, or 3 drug molecules per polymer chain (abbreviated P7D1, P7D2, P7D3). We hypothesize that analogues of these prodrugs naturally arise during partial degradation of P7D3. We found that varying the number of conjugated drug molecules generates precise control over drug dosing, without sacrificing the stability of assembled, supramolecular objects. We also demonstrate that such assembly is directly related to the ratio of DPCA to PEG. For the three prodrugs, we observe the transition from soluble prodrug via spherical micelles to worm-like micelles through wet- and dry-state imaging and scattering techniques, as well as coarse-grained modeling. In addition to influencing prodrug administration and handling, we explore the relationship between prodrug structure and mechanism of drug release, including partial hydrolysis of multidrug conjugates and susceptibility to enzymatic cleavage. Remarkably, we find that prodrug molecular architecture greatly influences drug binding and HIF-1a regulation. The results of this study highlight the importance of prodrug molecular architecture in determining bioactivity and performance of PEGylated drugs. We note that such prodrugs are rarely explored for applications other than cancer and emphasize the potential utility of DPCA in treating tissue damage brought on by trauma, age-related degeneration, and disease through the prodrug system developed here.

\section{Results and Discussion}

Prodrug Design, Synthesis and Purification. Fig. 1 outlines the molecular design and characterization of the three prodrugs analyzed in this study. Each prodrug consists of a linear monofunctional poly(ethylene glycol) (PEG) chain $(750 \mathrm{~g} / \mathrm{mol})$, functionalized with a mono-, di- or tri-hydroxy end group via an amide linker allowing for conjugation of 1, 2, or 3 molecules of DPCA. The rationale for our prodrug design stems from the hypothesis that intermolecular $\mathrm{H}$-bonding, van der Waals interactions, and pi-pi stacking interactions among 1,4-dihydrophenonthrolin-4-one-3-Carboxylic acid (DPCA) motifs directs the formation of supramolecular structures with a drug-rich core and a hydrophilic PEG corona that affords colloidal stability and prevents protein adsorption. With respect to drug delivery, the ester bonds of PEG-DPCA allow drug release via hydrolysis when exposed to aqueous environments in vivo. 
A

P7D1<smiles></smiles>

P7D2

\section{P7D3}
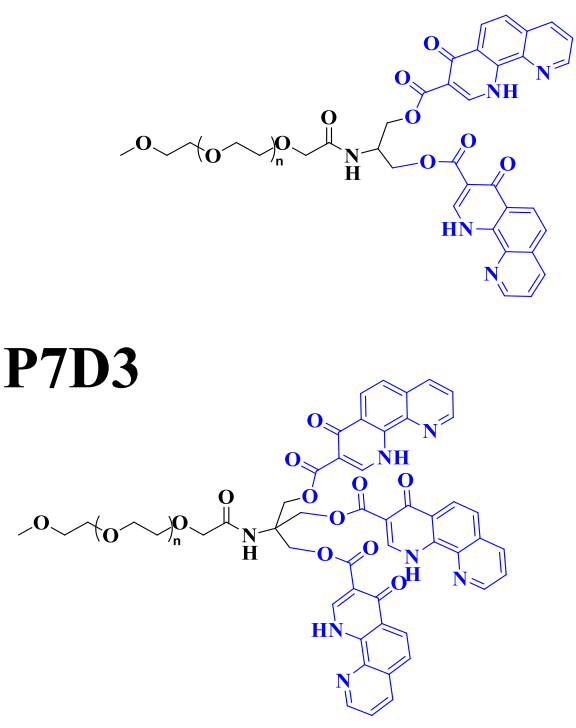
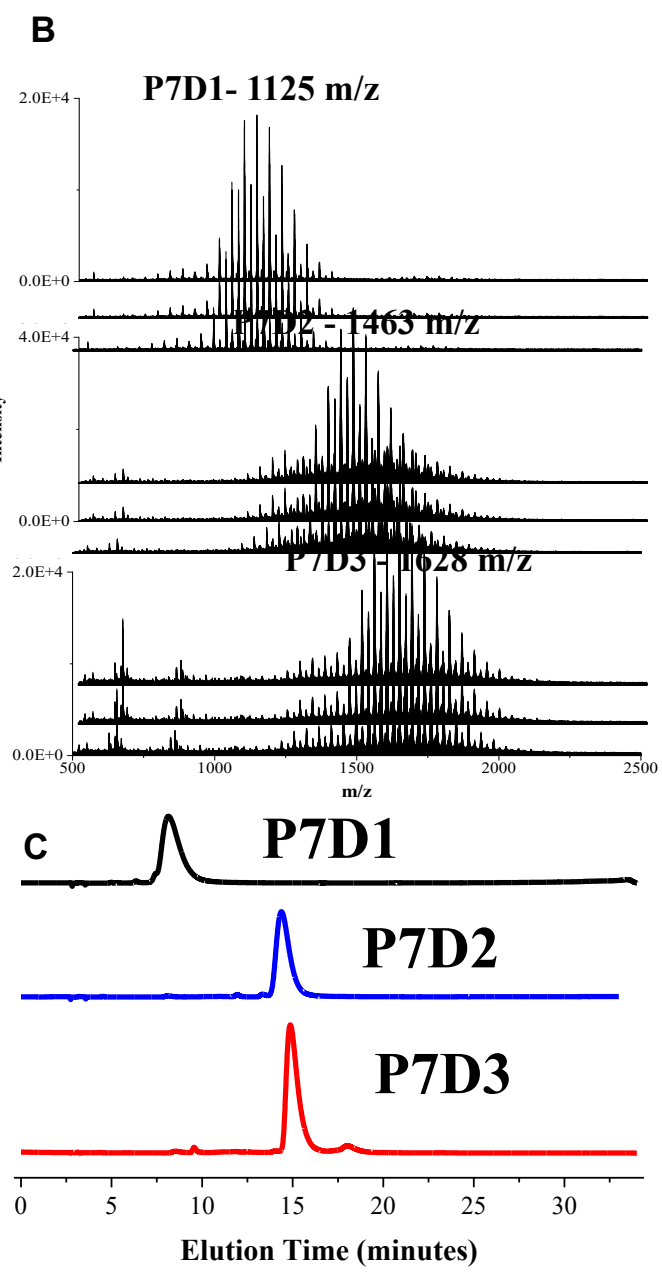

Figure 1. Molecular structures of PEG-based prodrugs P7D1, P7D2, and P7D3 are shown (A). In each case, DPCA (blue) is conjugated to a $750 \mathrm{~g} / \mathrm{mol}$, linear PEG (black), via a hydrolysable ester linkage. Successful drug coupling is verified by increasing molecular weight as seen in MALDI-TOFMS (B) and elution time in analytical HPLC (C). After purification, free DPCA and PEG, as well as partially coupled prodrugs are removed.

Detailed synthetic procedures for PEG-DPCA prodrug synthesis can be found in the Supplementary Information. Briefly, synthesis involves TEMPO-mediated oxidation of methoxy PEG followed by HBTUmediated coupling of 2-aminoethanol, 2-amino-1,3-propanediol, or 2-amino-2-(hydroxymethyl)-1,3propanediol to yield mono-, di-, or trihydroxy functional PEG (PEG-(OH) 1 , PEG-(OH) $)_{2}$ PEG-(OH $\left.)_{3}\right)$. DPCA was then conjugated to the terminal hydroxyls of the PEG chains via 1,1'-Carbonyldiimidazole-activated esterification to generate the prodrugs P7D1, P7D2 and P7D3 $\left({ }^{1} \mathrm{H}-\mathrm{NMR}\right.$ in Figure S5). Prior to characterization, crude reaction products containing partially coupled prodrugs or free, uncoupled DPCA were purified via semi-preparative HPLC fractionation or silica gel column chromatography. Final products were characterized via analytical HPLC and mass spectrometry showing a final prodrug purity of $>95 \mathrm{~mol} \%$ P7DX content.

Self-Assembly of PEG-DPCA Prodrugs. Self-assembly of PEG-DPCA prodrugs was achieved by direct dissolution of lyophilized powders from purification in water or PBS buffer at $50^{\circ} \mathrm{C}$ for 30 minutes. At this temperature, prodrugs are assumed to exist in a non-assembled, soluble state based off of solution-gel transition temperatures identified in previous work. ${ }^{6}$ After heating, a complete solution with no visible precipitates was obtained for all prodrugs. The solution was then cooled to room temperature $\left(25^{\circ} \mathrm{C}\right)$ for 1 
hour with slight agitation, before characterization. Rheological characterization of assembled samples showed that at high concentrations, P7D3 suspensions displayed a high-viscosity, shear-thinning behavior characteristic of entangled nanofiber solutions. However, this behavior was not observed in P7D1 and P7D2 samples, which instead exhibited shear-responses analogous to water, even at $100 \mathrm{mg} / \mathrm{mL}$ (Figure S7).

To determine if PEG-DPCA prodrugs formed stable, supramolecular aggregates beyond a critical micelle/fiber concentration (CMC/CFC) a Nile Red probe assay was employed ${ }^{30,31}$. While the fluorescence of Nile Red is quenched in water, it emits strongly in hydrophobic environments when excited at $490 \mathrm{~nm}$. Thus, partition of the dye into the hydrophobic, DPCA-rich core during self-assembly of prodrug structures, is likely to be captured by a non-linear increase in Nile Red fluorescence with prodrug concentration. This effect was observed for all PEG-DPCA conjugates, suggesting aggregation above a critical concentration in the range of $1 \mathrm{mM}$. This value is in good agreement with PEG-phenanthroline compounds reported by Nagy et. al. ${ }^{32,33}$ Interestingly, the CMC values for PEG-DPCA assemblies do not seem to be directly related to DPCA content (Table 1). This is surprising, as the hydrophobic nature of DPCA is presumed to be the main driving force for self-assembly. We speculate that this result does not arise due to limitations in our experimental technique, as we were able to capture accurate CMC values for common surfactants SLS and Triton-X at lower concentrations (Figure S8). The observed trend showing the lowest CMC value for P7D2, followed by P7D1 and P7D3 may instead be influenced by prodrug geometry thus highlighting an important structure-property relationship. Possibly, the stereochemical limitations for rotation within the spacer molecules for 1, 2, and 3 DPCA units can act as a limiting factor for self-assembly. Thus, assembly into objects of different shapes may occur at the same concentration. It is possible that for both P7D2 and P7D3 prodrugs, the presence of intramolecular interactions between DPCA molecules on the PEG chain, increase prodrug solubility and $\mathrm{CMC}$, despite their large, hydrophobic volume fraction as compared to P7D1. This phenomenon would also explain the higher CMC for P7D3 when compared to P7D2.

Table 1: Critical Micelle Concentration values for polymer prodrugs with 1, 2, or 3 DPCA per PEG.

\begin{tabular}{|l|l|c|}
\hline \multicolumn{1}{|c|}{ Prodrug Structure } & Average CMC (P7DX mg/ml) & Average CMC (P7DX mM) \\
\hline P7D1 & $2 \pm 0.5 \mathrm{mg} / \mathrm{mL}$ & $1.85 \mathrm{mM}$ \\
\hline P7D2 & $1 \pm 0.2 \mathrm{mg} / \mathrm{mL}$ & $0.73 \mathrm{mM}$ \\
\hline P7D3 & $3.2 \pm 0.7 \mathrm{mg} / \mathrm{mL}$ & $2.1 \mathrm{mM}$ \\
\hline
\end{tabular}

After indirectly verifying self-assembly using the Nile Red probe assay, the morphology of PEG-DPCA supramolecular structures was visualized through both dry and wet-state imaging of aqueous suspensions above CMC. In Fig. 2A-B, micrographs obtained from TEM, show predominately spherical, micelle-like structures for P7D1 and P7D2 with average diameters of $15 \mathrm{~nm}$ and $27 \mathrm{~nm}$, respectively (Figure S9). With estimated PEG chain length ranging from $4.7 \mathrm{~nm}$ and $6.1 \mathrm{~nm}^{34}$, and DPCA head group area of approximately $0.2 \mathrm{~nm}^{3}, 0.5 \mathrm{~nm}^{3}$, and $0.9 \mathrm{~nm}^{3}$ for P7D1, P7D2, and P7D3 respectively, this nanoaggregate geometry and size are in good agreement with our theoretical predictions. DLS measurements on aqueous solutions at the same concentrations reported larger hydrodynamic diameters for P7D1 and P7D2 of $40 \mathrm{~nm}$ and $87 \mathrm{~nm}$, likely due to swelling of wet aggregates (Figure S10). Dispersity in particle size observed in both TEM and DLS likely arise from the high dispersity in PEG molecular weight (Fig. 1B) rather than differences in self-assembly behavior. 
A

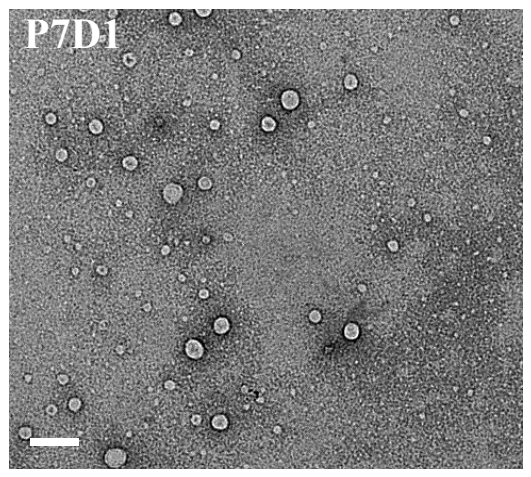

B

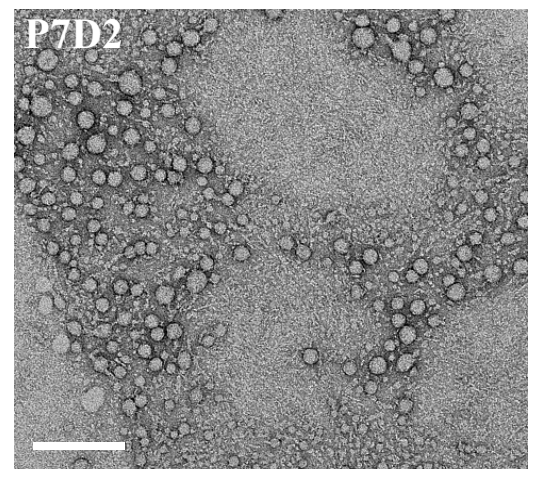

C

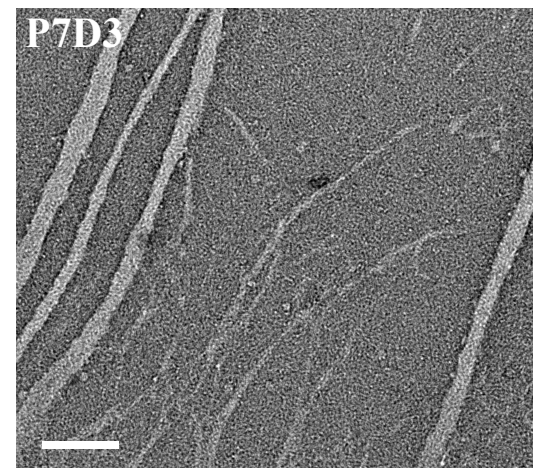

Figure 2. TEM micrographs of PEG-DPCA prodrugs, prepared from $7 \mathrm{~g} / \mathrm{L}$ aqueous dispersions. For P7D1 (A) and P7D2 (B) samples, spherical assemblies were observed. For P7D3 (C), single fibers and bundles were observed. All selfassembled structures presumably consist of a DPCA-rich core and PEG corona. Scale bar $=200 \mathrm{~nm}$

When the number of DPCA molecules per PEG chain is increased to 3, worm-like micelles were observed in TEM (Fig. 2C). Image analysis suggests that the supramolecular fibers measure several microns in length and $16 \mathrm{~nm}$ in width. DLS analysis of P7D3 indicated a hydrodynamic diameter of $228 \mathrm{~nm}$ from DLS, which is likely inaccurate due to spherical assumptions used in Stokes Einstein equation when estimating diameter (Figure S10). While the dominating population observed in TEM was found to be worm-like micelles, spherical aggregates were visible in several samples (Figure S11). To corroborate findings from dry-state imaging with wet-state characterization, SAXS analysis was performed at $25 \mathrm{~g} / \mathrm{L}$ to generate information on assembled state, distribution, and size of the scattering domains. The 1D plot of the samples shown in Figure S13 indicates a strong structural dependence on the objects depending on the number of DPCA units, all deviating from a PEG reference solution. Despite the difficulty in fitting the curves to a specific geometry, explained also by the distribution of structures seen in EM-images and noted in Figure 2, the trend seems to be that P7D3 forms domains of smaller size but larger distributions (slope in the qrange at 0.1 ) and P7D2 show large sizes, further corroborating the suspected wet-state assembly and strengthening the finding that altering the number of DPCA units, significantly affects carrier morphology.

The biodistribution of drug delivery vehicles is known to be influenced by size and morphology. For example, worm-like micelles have generally been found to be more resistant to phagocytic clearance and exhibit longer circulation times than their spherical counterparts due to differences in their response to shear forces and higher aspect ratio ${ }^{18,35}$. Amongst spherical particles, those measuring 20 to $150 \mathrm{~nm}$ in diameter are generally considered to be resistant to rapid renal clearance but are still able to navigate physiological barriers without activating the complementary system ${ }^{36}$. Thus, the array of supramolecular objects developed here, may have differential utility when targeting specific injuries. In addition to altering biodistribution post-administration, nanostructure morphology also directly informs delivery route. At high concentrations, worm-like micelles formed from P7D3 creates a viscous, shear-thinning gel, currently being explored for subcutaneous and topical delivery of DPCA (Figure SX). However, P7D1 and P7D2 micelle suspensions, show Newtonian behavior similar to water or low molecular weight PEG solutions even at 100 $\mathrm{mg} / \mathrm{mL}$. Therefore, these materials will likely be more suitable for intravenous delivery to systemic uptake of drug. In future work, it may also be possible to decorate the surfaces of the supramolecular assemblies with tissue-specific epitopes for directing targeting.

Molecular Dynamics Simulations. We argue that, in accordance with traditional amphiphile assembly pathways $^{37}$, PEG-DPCA self-assembly is driven by two key parameters: molecular geometry and the contrasting solvent affinities of hydrophilic PEG and hydrophobic DPCA. To interrogate these driving forces, 
we conduct coarse-grained molecular dynamics simulations that capture the salient intermolecular interactions and structural features of P7D1, P7D2, and P7D3. Unlike commonly examined amphiphiles, the PEG-DPCA polymer-drug conjugates feature a flexible hydrophilic component (PEG) and a bulky hydrophobic component (DPCA). Employing an implicit solvent model, we account for these contrasting solvent affinities with different interaction potentials. Each DPCA molecule is represented as a single bead that is attracted to other DPCA beads, and the PEG chain is modeled as linearly connected beads-on-aspring that repel all the other beads through excluded volume interactions. The intramolecular DPCA beads were arranged to match the lowest energy geometry identified through the MM2 force field, a commonly applied force field for determining conformations of hydrocarbon chains and small organic molecules ${ }^{38}$. A rigid body constraint was applied to fix the relative positions of the DPCA beads and the connecting PEG bead $^{39}$. Based on these calculations, the lone DPCA bead in P7D1 lies in the same plane as the polymer chain, with an angle of $180^{\circ}$ between the DPCA and the connecting PEG bead. The 2 DPCA beads in P7D2 form an equilateral triangle with the connecting PEG bead. The 3 DPCA beads in P7D3 form a tripod with the connecting PEG bead (Fig. 3A).

Simulations were run for volume fractions $0.002<\phi<0.008$ and DPCA attraction strengths $5 k_{B} T<\varepsilon_{A}<15$ $k_{B} T$ (see Methods for precise definitions of $\phi$ and $\varepsilon_{A}$ ). The $\phi$ range was selected to explore concentrations at which micelles and fibers have been observed experimentally. The $\varepsilon_{A}$ range was chosen based on the range of DPCA-DPCA attraction strength found in DSC measurements of the enthalpy of fusion of pure DPCA. Equilibrated simulations (equilibration details provided in Supplemental) result in a variety of selfassembled structures featuring a DPCA core surrounded by a PEG corona (see Fig. 3D-F inset).

For a DPCA attraction strength of $\varepsilon_{A}=12.5 k_{B} T$ (the approximate experimental value at room temperature [see DSC Figure S6] increasing the DPCA content of the amphiphile results in fewer clusters forming per volume (Fig. 3B) but larger clusters (Fig. 3C) at all but the lowest density examined. Moreover, the concentration dependence of the cluster density and mean cluster size is qualitatively distinct for the P7D3 compared to the P7D1 and P7D2 systems. The size of P7D3 clusters increases linearly with concentration, while the cluster density only increases sublinearly. In contrast, P7D1 and P7D2 both exhibit a sublinear increase in mean cluster size but a nearly linear increase in cluster density (Fig. 3C). The disparate scaling of cluster density and cluster size for P7D3 compared to P7D1 and P7D2 points to a morphological difference in the self-assembled aggregates resulting from topological differences in the amphiphiles due to the number of drug molecules linked to the polymer chain. 
A
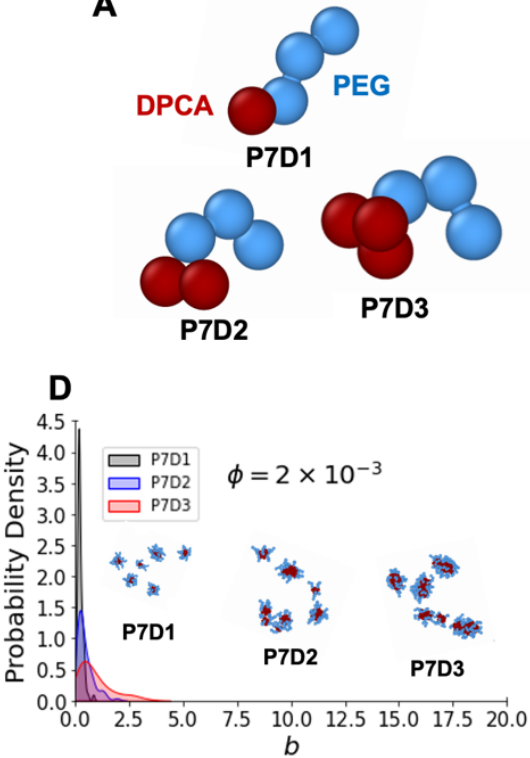

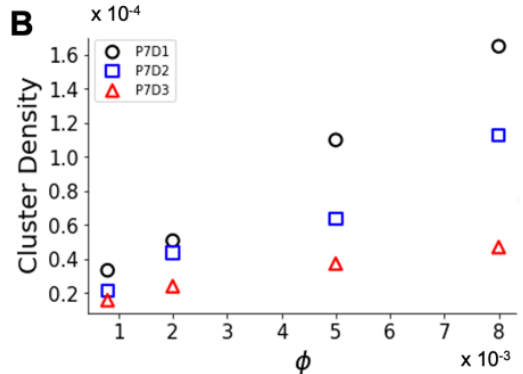

E

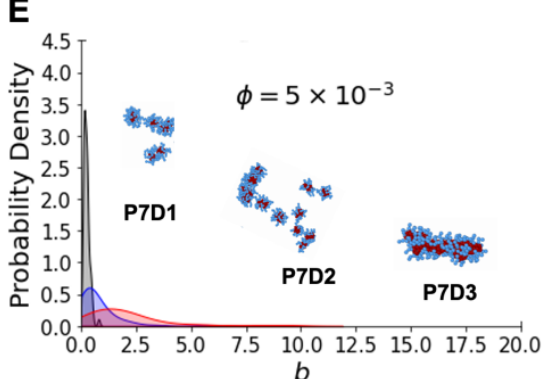

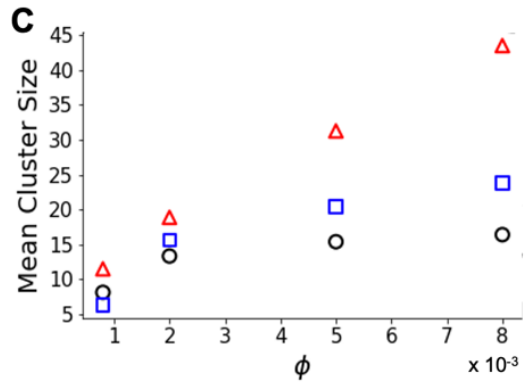

$\mathbf{F}$

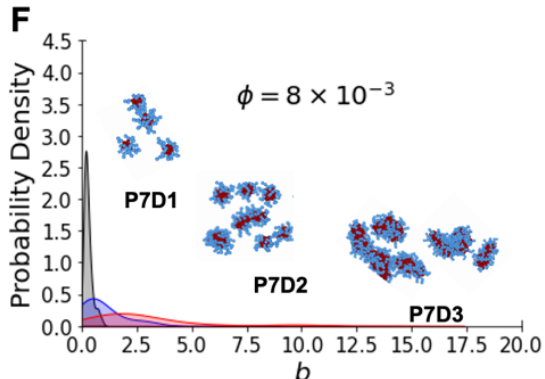

Figure 3. Increasing number of DPCA leads to aggregates with more fiber-like geometries. A) coarse-grained representations of P7D1, P7D2, and P7D3 featuring a flexible polymer chain (blue) appended to 1, 2, or 3 DPCA molecules (red); B) dependence of cluster density (in units of $\sigma^{-3}$; see Methods for definition of $\sigma$ ) on volume fraction; $C$ ) dependence of mean cluster size (number of molecules per cluster) on volume fraction; $D-F$ ) Asphericity distributions $b$ (in units of $\sigma$ ) at volume fractions $\phi=0.002,0.005,0.008$. Simulation snapshots depict spherical micelles formed by $P 7 D 1$, elongated micelles formed by P7D2, and fibers formed by P7D3. All data is presented at attraction strength $\varepsilon_{A}$ $=12.5 k_{B} T$.

To further explore these morphological differences, we analyzed the shapes of the self-assembled aggregates by computing the asphericity parameter $b$ for each cluster (see Methods for details, including the nondimensionalization of $b$ ). For perfectly spherical clusters, $b=0$. Larger values of $b$ indicate less spherical morphologies, such as fiber-like aggregates ${ }^{40}$.

Analyzing the probability density of $b$, we found that the asphericity of P7D1 clusters never exceeds a value of $b=1.0$, even at densities nearly ten times higher than the experimentally observed CMC (this corresponds to $\phi=0.008$ in our simulations, see Table S1 for conversion). P7D2 clusters are slightly less spherical, reaching $b=3.0$ for some clusters at $\phi=0.005$ and $b=5.0$ for clusters at $\phi=0.008$.

The asphericity distribution of P7D3 exhibits a striking departure from those of P7D1 and P7D2 (see Figure S14). At the same densities, P7D3 self-assembles into highly aspherical structures, with the probability density exhibiting a long tail that reaches values as high as $b=17.5$ when $\phi=0.008$. Our simulations confirm the presence of fiber-like structures for P7D3 at densities and attraction strengths where P7D1 and P7D2 are limited to spherical micelles. This finding corroborates the experimental evidence that suggests only P7D3 forms nanofibers at the conditions examined. Future work will examine the precise role of the topology of the hydrophobic group in the phase behavior of these systems.

In vitro Drug Release and Hydrolysis. Our long-term vision for PEG-DPCA is to induce tissue regeneration in non-healing mammals by upregulation of HIF-1 $1 \alpha$ via controlled delivery of DPCA. Thus, we aim to capitalize on the modularity of our initial prodrug design to develop new carriers capable of delivering drug through different administration routes and to diverse target locations. Therefore, after elucidating the relationship between prodrug molecular design and self-assembly, we investigated the performance of P7D1, P7D2 and P7D3 as drug delivery vehicles. As seen in Fig. 4, the kinetics of this event are directly related to prodrug molecular design and supramolecular morphology. Although release of DPCA is presumed to occur through hydrolysis of the PEG-DPCA ester linkage for all prodrugs investigated, two separate regimes were considered: below $\mathrm{CMC}$ and above CMC. 
Below CMC. For soluble (unassembled) polymer prodrugs, the effects of increasing the number of DPCA units at the PEG chain end were observed by monitoring hydrolysis of prodrug solutions below CMC (Fig. 4A). Compared to P7D3, which shows little to no hydrolysis over 5 weeks $(<2 \%)$, hydrolysis of P7D2 and P7D1 appears to result in near zero-order release kinetics. At the conclusion of 5 weeks, hydrolysis of approximately $10 \%$ of conjugates has been observed. Significant differences in P7D3 and P7D1/P7D2 stability can likely be attributed to strong intramolecular interactions between DPCA molecules, creating an increasingly hydrophobic environment as the number of DPCA per PEG is increased from 1 to 3 . HPLC and MALDI-MS analysis of P7D3 incubated in water revealed the emergence of partially hydrolyzed PEGDPCA as well as free PEG (Figure S15), suggesting that DPCA release may occur in a stepwise fashion through P7D2 and P7D1 intermediates, before complete drug release is achieved.
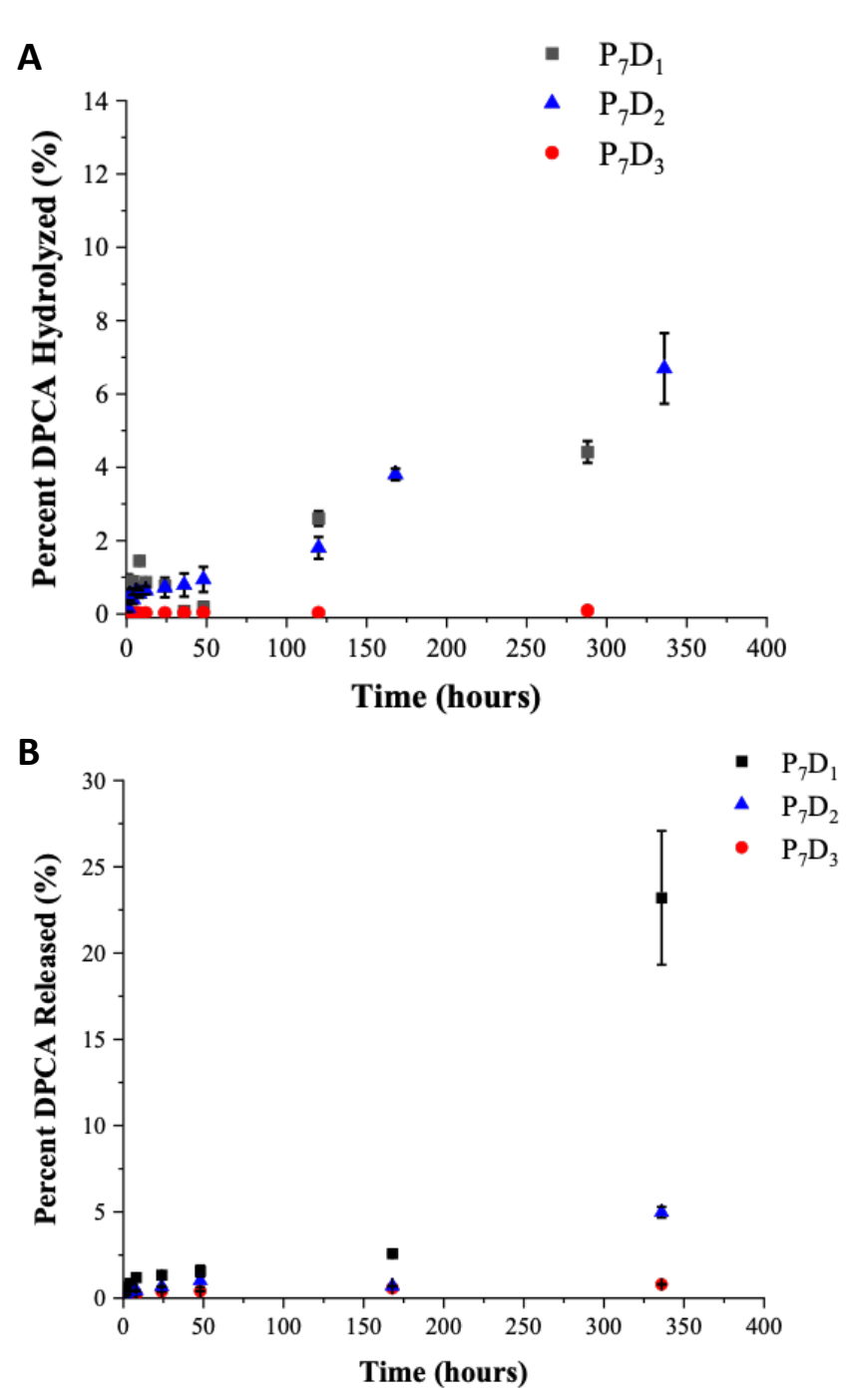

\section{C}
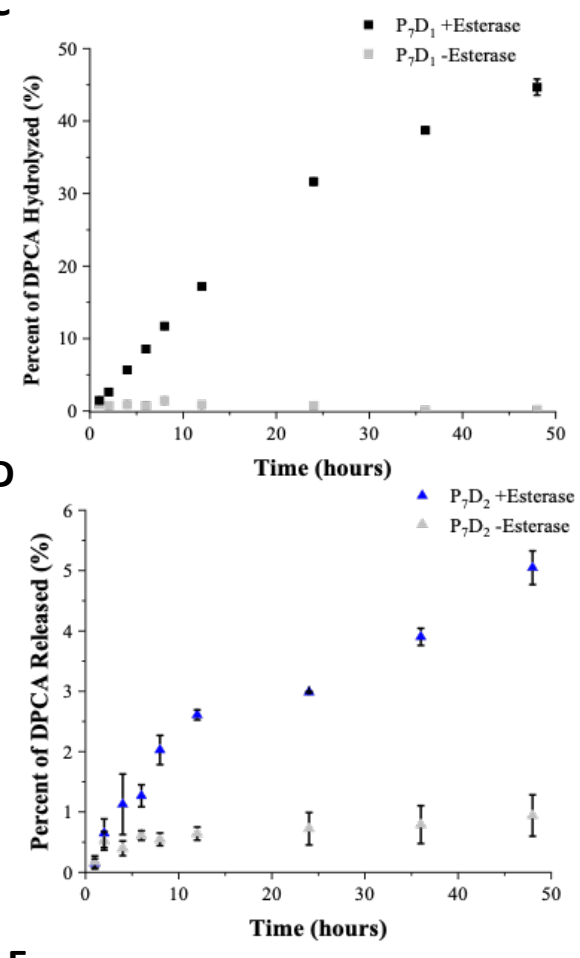

E

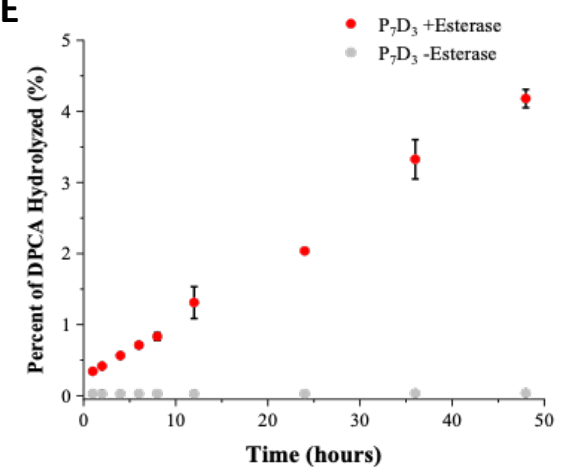

Figure 4. Drug release from PEG-DPCA prodrugs was measured in the non-assembled (A) and assembled state (B). Overall, at neutral $\mathrm{pH}$ and $37^{\circ} \mathrm{C}$, the release kinetics is extremely slow. In the presence of esterase enzymes, however, release is drastically accelerated for all prodrugs (C-E). Overall, the kinetics of DPCA release are directly proportional to prodrug hydrophobicity.

Above CMC. Above CMC (in the self-assembled state), DPCA release was significantly slower for all prodrugs, with only $2 \%$ of drug released over 2 weeks (Fig. 4B). This slower rate is likely due to decreased exposure of the DPCA ester linkage to water within the hydrophobic core of assembled structures. While 
slow, the kinetics of drug release again appear to be inversely related to DPCA content. Thus, by modulating the molecular design of prodrugs, we are not only able to precisely direct self-assembly, but control drug dosing and release for specific applications.

Our previous in vivo study using hydrogels based on P7D3 showed that upregulation of HIF-1a occurred as soon as one day after administration ${ }^{6}$. This response suggests that the rate of drug release in vivo, may be much more rapid than that observed in the in vitro hydrolysis and release studies. To better understand this discrepancy, we next sought to determine if PEG-DPCA prodrugs are susceptible to enzymatic hydrolysis by carboxyl esterase enzymes, commonly found in mammalian liver cells and blood plasma. As seen in Fig. 4C-E, following incubation with porcine liver esterase (EC 3.1.1.1), DPCA release is significantly increased P7D1, P7D2 and P7D3 solutions below CMC. While, increasing DPCA conjugation does not prohibit substrate recognition, the relationship between release kinetics and prodrug molecular structure is still preserved with the order of absolute drug release as follows: P7D1 > P7D2 > P7D3. We note that P7D1 shows significant drug hydrolysis (>30\%) after 24 hours, while P7D2 and P7D3 only reach $5 \%$ hydrolysis in the presence of esterase. We speculate that this effect can be attributed to prodrug geometry, and that the higher density of DPCA in P7D2 and P7D3 reduces substrate recognition and interactions with the esterase active site.

Prodrug Bioactivity. Finally, the ability of prodrug (P7D1, P7D2 and P7D3) to stabilize HIF-1a was evaluated in primary human amnion endothelial cells (AECs). AECs make up a major component of the fetal membrane, which is incapable of repairing defects that may be brought on by fetoscopic surgery and is therefore a promising candidate for novel regenerative therapies ${ }^{41}$. AECs were treated with soluble prodrugs (below CMC) and identical DPCA concentrations of $60 \mu \mathrm{g} / \mathrm{mL}$. We observed that after 4 hours, AECs treated with conditioned media containing P7D3 displayed robust expression of HIF-1a, limited expression following P7D1, and no obvious stabilization in the case of P7D2, as compared with free DPCA (Figure 5). DPCA is claimed to act as a competitive inhibitor of the 2-oxoglutarate cofactor that is needed for hydroxylation of HIF-1 $\alpha^{12,13,15}$. However, DPCA is likely capable of ligating to the active iron site within PHD enzymes via the phenanthroline and/or carboxylic acid moieties ${ }^{15,33}$. Computational molecular docking of DPCA against HIF hydroxylases has predicted that bidentate iron ligation via the carboxylic acid moiety is preferred and analogues of DPCA lacking one nitrogen or one pyridine ring exhibit reduced activity ${ }^{14}$. To investigate the iron-binding behavior of P7D1, P7D2 and P7D3, iron(II) (FeCl2 (aq)) was added at 1:3 molar ratio to DPCA. and UV-vis spectra recorded. Color changes were suggestive of iron binding (Figure S16), although significant differences were noted for the iron binding spectra of DPCA, P7D1, P7D2 and P7D3. It is possible that differences in iron binding and bioactivity of PEG-DPCA prodrug may be related to the molecular architecture of the prodrugs, however the mechanism of PHD inhibition is known to be complex, ${ }^{42}$ and further studies will be needed to investigate the mechanism of PEG-DPCA inhibition of PHD. 


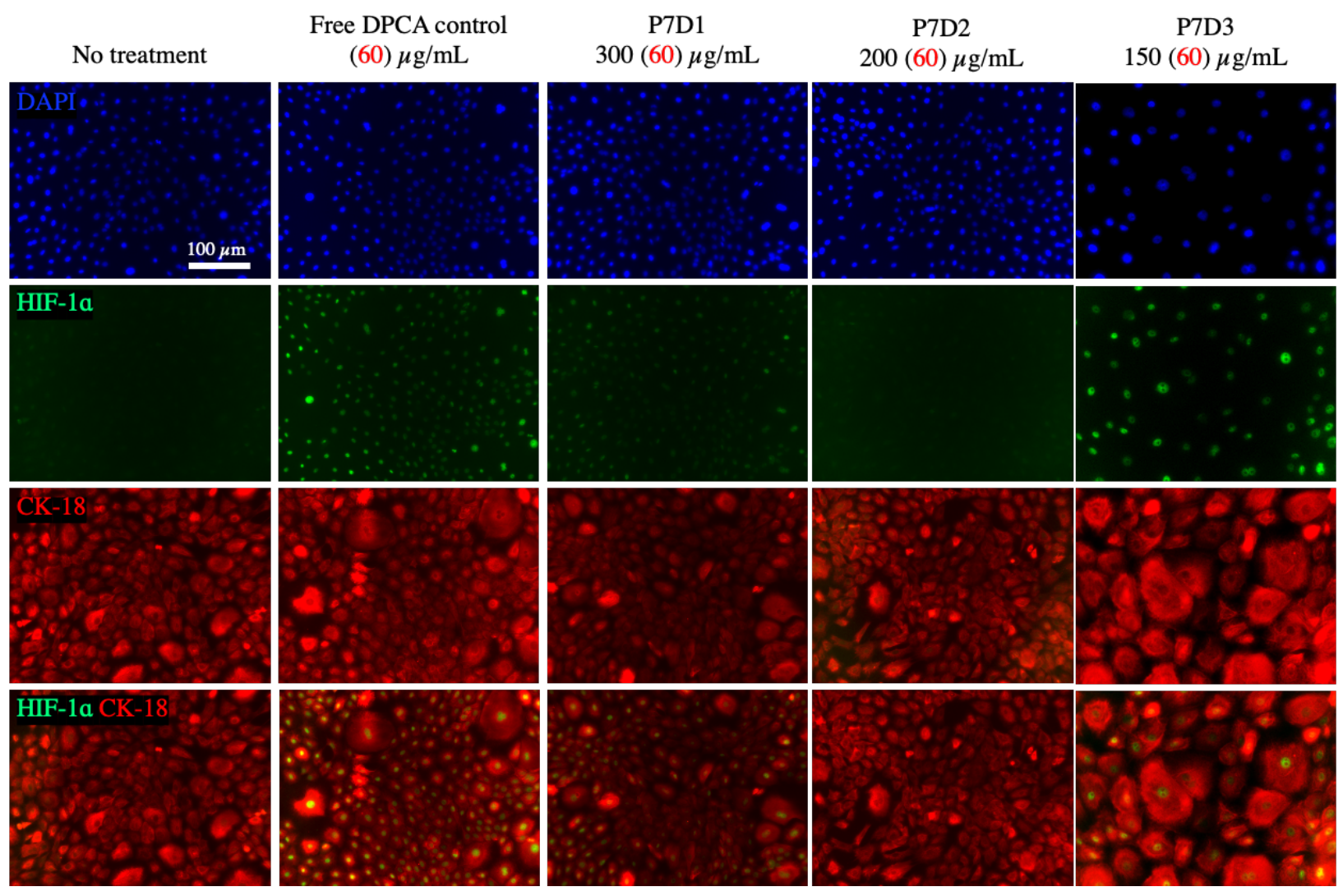

Figure 5. Transient stabilization of HIF-1a in human amnionic endothelial cells treated with growth media containing free DPCA or PEG-DPCA prodrugs for 4 hours.

\section{Conclusion}

The utility of macromolecular prodrugs, created by covalently bonding bioactive compounds to polymers or peptides is well known. Rationally designing these prodrugs to achieve desired biodistribution profiles, release kinetics, and activity, however, remains a significant challenge. In this study, we elucidated the structure-property relationship of a PEG-based prodrug created to deliver the pro-regenerative drug, DPCA. Our general design involved covalently bonding DPCA, a poorly water-soluble PHD inhibitor to the terminal end of a linear PEG via hydrolysable ester bonds. By controlling drug conjugation per PEG chain, we were able to precisely direct self-assembly behavior to form supramolecular nanostructures visualized in the wetand dry-state, confirming a significant dependency on prodrug molecular design and drug carrier performance. Differences in the ability of PEG-DPCA prodrugs to stabilize HIF-1 $\alpha$ in human cells suggest a complex relationship between molecular architecture and PHD inhibition. Fundamentally, this study highlights the importance of understanding the relationship between molecular design, self-assembly, prodrug stability and bioactivity when designing supramolecular polymer prodrugs. Since HIF-1a is expressed in all cell types, we speculate that the translational potential of these prodrugs will be of interest for many applications in regenerative medicine.

\section{Acknowledgements}

The authors would like to thank Dr. Tomoko Ogiyama and Erika Salzman for contributions to DPCA and Im-DPCA synthesis, and Dr. Jaffar Hasnain and Gautam Bordia for helpful discussions. SAXS 
experiments were performed at Beamline 7.3.3 of the Advanced Light Source at Lawrence Berkeley National Laboratory. The Advanced Light Source is supported by the Director, Office of Science, Office of Basic Energy Sciences, of the U.S. Department of Energy under Contract No. DE-AC02-05CH11231. Thanks also to Dr. Andrew Jimenez for SAXS data processing and Dr. Chenhui Zhu for data collection. This work was supported by the National Science Foundation Graduate Research Fellowship under Grant No. DGE 1752814; the National Institutes of Health Grants RO1DE021104 and R01EB022031; and US Department of Defense Grant W81XWH1910468. We acknowledge NIH grant S10OD024998 for funding of the AV-600 $\mathrm{MHz}$ NMR instrument.

\section{Materials and Methods}

Details on synthesis and additional experimental protocols can be found in the Supplementary Information.

Preparation of Self-Assembled PEG-DPCA Structures. Lyophilized powders of PEG-DPCAs obtained from semi-preparative fractionation were added directly to ultrapure water or $10 \mathrm{mM}$ PBS buffer $(\mathrm{pH} 7)$. Solutions were then heated to $50^{\circ} \mathrm{C}$ in a water bath for 30 minutes, with periodic vortexing. Fully soluble, homogeneous solutions were then removed from heat and cooled to $25^{\circ} \mathrm{C}$ in a bench-top shaker table for 1 hour. Prior to dilution, all PEG-DPCA solutions were always re-heated to $50^{\circ} \mathrm{C}$ and cooled.

CMC Measurements. $0.5 \mathrm{uL}$ of $2.5 \mathrm{mM}$ Nile Red stock solution prepared in ethanol was added to a microcentrifuge tube. Tubes were then shielded from light and left open to allow ethanol to evaporate overnight. $500 \mathrm{uL}$ of aqueous PEG-DPCA suspensions at various concentrations or control solutions were then added to each tube. To promote interactions between dye and self-assembled structures, mixtures were vortexed rigorously and placed on a bench-top shaker overnight, protected from light. $150 \mathrm{uL}$ of each sample were then transferred to a black 96 well-plate and emission spectra were collected from 520 to 700 $\mathrm{nm}$ at $490 \mathrm{~nm}$ excitation using a plate reader (Biosystems Synergy H1 Microplate Reader) and compared to background samples containing no dye. Spectra were normalized to non-dye controls and peak fluorescence intensity at $670 \mathrm{~nm}$ was extracted and graphed versus log of prodrug concentration. The CMC was calculated as the intersection of two lines of best fit for the lower and upper regions of data. Standard deviation was determined from at least 2 experimental replicates.

TEM. Aqueous suspensions of self-assembled PEG-DPCA conjugates prepared at $7 \mathrm{~g} / / \mathrm{L}$ were drop-casted onto carbon-coated copper grids (Electron Microscopy Sciences, CF400-Cu). After 5 minutes, excess liquid was removed by blotting with filter paper. For stained samples, a $1 \%$ uranyl acetate aqueous solution was added to the grid for 3 minute and blotted with filter paper. TEM images were taken on a FEI Tecnai 12 transmission electron microscope (Electron Microscopy Lab, UC Berkeley).

DLS Measurements. The hydrodynamic radius $\left(\mathrm{D}_{\mathrm{H}}\right)$ and polydispersity index (PDI) of the self-assembled PEG-DPCAs was determined with a Malvern Zetasizer NanoZS at $25^{\circ} \mathrm{C}$ at concentrations of $7 \mathrm{mg} / \mathrm{mL}$ in $\mathrm{H}_{2} \mathrm{O}$. For DLS measurements, warmed solutions were filtered through a $0.22 \mu \mathrm{M}$ syringe filter prior to cooling.

Coarse-Grained Modeling. We represent each DPCA molecule as a single bead. Intermolecular DPCADPCA interactions are modeled with a Lennard-Jones (LJ) 6-12 potential that is cut off at $2.5 \sigma$ (where $\sigma$ is the LJ diameter) to capture the attraction between DPCA molecules. The strength of DPCA-DPCA attractions is set by the LJ well depth, $\varepsilon_{A}$, a key control parameter in our study. The $750 \mathrm{Da} P E G$ is represented as three linearly connected LJ beads (with the same $\sigma$ as the DPCA beads) using the KremerGrest model ${ }^{43}$. This choice results in an average end-to-end distance of our coarse-grained PEG of $R_{\mathrm{ee}}=$ $1.88 \sigma \pm 0.003 \sigma$. The ratio of $R_{\text {ee }}$ to the DPCA size $\left(2^{1 / 6} \sigma\right)$ is then 1.67 , which is nearly identical to the experimentally estimated value of 1.68 by Chen et. al ${ }^{6}$. The PEG-DPCA and PEG-PEG LJ interactions are cut off at $2^{1 / 6} \sigma$ to model purely repulsive interactions with the Weeks-Chandler-Anderson potential ${ }^{44}$. The $\mathrm{LJ}$ well depth of these repulsive interactions is set to the thermal energy $k_{B} T$. 
All simulations were conducted using the GPU-enabled opensource HOOMD-blue simulation package ${ }^{45}$. Each simulation consists of 1000 molecules and was equilibrated using an annealing procedure described in the Supplemental. Simulations were performed in a cubic box for volume fractions $0.002<\phi<0.008$ and attraction strengths $5 k_{B} T<\varepsilon_{\mathrm{A}}<15 k_{B} T$ as this is the estimated range at which micelles and fibers were experimentally observed. The volume fraction $\phi$ is defined as the ratio of the solute volume (the product of the number of beads $N_{B}$ and the volume of each bead $V_{B}$ ) to the total system volume $V_{\text {sys }}$ i.e., $\phi=N_{B} V_{B} / V_{\text {sys }}$. For the spherical bead volume, we take the bead diameter to be $2^{1 / 6} \sigma$, the distance below which all particle pairs repel each other.

Simulations were analyzed using the open-source simulation and visualization software OVITO ${ }^{46}$. Beads within a cutoff distance of $1.2 \sigma$ are grouped into the same cluster. The cluster density reported in Figure 3B was calculated by dividing the number of clusters by the system volume. The mean cluster size reported in Figure $3 \mathrm{C}$ was determined by averaging the number of molecules in a cluster. We chose molecules rather than beads to facilitate comparisons between the assembly of molecules with different DPCA contents.

The asphericity $b$ of each cluster was then computed by diagonalizing its gyration tensor $S$

$$
\boldsymbol{S}=\left[\begin{array}{ccc}
\lambda_{x x} & 0 & 0 \\
0 & \lambda_{y y} & 0 \\
0 & 0 & \lambda_{z z}
\end{array}\right]
$$

following the convention $\lambda_{z z}>\lambda_{y y}>\lambda_{x x}$, (note that $\lambda$ has units of length squared). The asphericity parameter $b$ is the difference between the longest component and the average of the two smaller components.

$$
b=\lambda_{z z}-\frac{1}{2}\left(\lambda_{x x}+\lambda_{y y}\right) .
$$

All asphericities reported are in units of the LJ bead diameter $\sigma$.

Hydrolysis and Enzymatic Cleavage of PEG-DPCAs. Solutions of PEG-DPCAs were prepared in PBS buffer following the standard protocol outlined above. At $t=0$, a $10 \mathrm{mg} / \mathrm{mL}$ stock solution of esterase from porcine liver (Sigma Aldrich, Cat\# 9016-18-6) was added to +Enzyme samples to achieve a final prodrug concentration of $0.3 \mathrm{mM}$ and enzyme concentration of $1 \mathrm{mg} / \mathrm{mL}(28 \mathrm{U} / \mathrm{mg})$. -Enzyme samples were diluted to the final prodrug concentration using PBS buffer. Samples were then vortexed, and placed in a temperature-controlled, bench-top shaker set to $37^{\circ} \mathrm{C}$. At predetermined timepoints, concentration of free DPCA and PEG-DPCA was determined using an Agilent 1260 Infinity HPLC in analytical mode with UV detection at $261 \mathrm{~nm}$ and gradient mobile phase from $70 / 30 \mathrm{v} / \mathrm{v} \% \mathrm{H}_{2} \mathrm{O} /$ Acetonitrile (ACN) with $0.1 \%$ TFA to $100 \%$ ACN over 30 minutes.

DPCA Release from Self-Assembled PEG-DPCAs. $6.4 \mathrm{mM}$ solutions of PEG-DPCAs were prepared in PBS buffer following the standard protocol outlined above. At $t=0,0.5 \mathrm{~mL}$ of cooled suspensions were loaded into dialysis cassettes with $2 \mathrm{k} \mathrm{MW}$ cutoff. Cassettes were then submerged in a $60 \mathrm{~mL}$ PBS buffer bath, sealed, and placed in a temperature-controlled, bench-top shaker set to $37^{\circ} \mathrm{C}$. At predetermined timepoints, a $1 \mathrm{~mL}$ aliquot was removed from the bath and replaced with fresh PBS. The concentration of DPCA from bath samples was determined using an Agilent 1260 Infinity HPLC in analytical mode with UV detection at $261 \mathrm{~nm}$ and gradient mobile phase from $70 / 30 \mathrm{v} / \mathrm{v} \% \mathrm{H}_{2} \mathrm{O} /$ Acetonitrile (ACN) with $0.1 \%$ TFA to $100 \%$ ACN over 30 minutes.

Cell Culture. Human amnion epithelial cells (hAECs) were isolated from the placental fetal membranes of patients undergoing elective caesarean delivery at UCSF Moore Women's Hospital. The fetal membranes were deidentified and considered as discarded human specimens exempt from IRB approval. After multiple washes with sterile ice-cold Hank's balanced salt solution (HBSS; Gibco) supplemented with 1\% penicillin/streptavidin (Gibco) and 0.1\% amphotericin B (Gibco), the amnion layer of the fetal membranes 
was mechanically separated from the chorion layer and cut into $1 \times 1 \mathrm{~cm}$ pieces. The amnion pieces were then transferred into $50 \mathrm{~mL}$ conical tubes containing $20 \mathrm{~mL}$ of prewarmed $0.25 \%$ trypsin/EDTA (Gibco) and incubated at $37^{\circ} \mathrm{C}$ for 10 min with gentle shaking. The obtained cells at this step were discarded to exclude blood clots and cellular debris. The remaining amniotic membrane pieces were transferred into new tubes containing trypsin/EDTA and incubated at $37^{\circ} \mathrm{C}$ for $30 \mathrm{~min}$ with gentle shaking for the second digestion. After neutralizing the enzymatic digestion with complete culture media [DMEM/F12 (Gibco) supplemented with $10 \%$ fetal bovine serum (FBS; Gibco), $1 \%$ penicillin/streptavidin, $0.1 \%$ amphotericin B and $10 \mathrm{ng} / \mathrm{mL}$ epidermal growth factor (EGF; PeproTech)], the solution was filtered through a 100- $\mu \mathrm{m}$ cell strainer and centrifuged at $200 \times \mathrm{g}$ for $5 \mathrm{~min}$ at $4^{\circ} \mathrm{C}$. The obtained cell pellets were re-suspended with the complete media and cultured in a tissue culture plate.

In vitro Bioactivity of PEG-DPCA. The day before PEG-DPCAs treatment, hAECs were seeded in a 96well plate at a density of $2 \times 10^{5} \mathrm{cells} / \mathrm{mL}$. The following day, solutions of PEG-DPCAs prepared by dissolving each PEG-DPCA powder in prewarmed complete media at predetermined concentrations. Solutions were then sterile filtered using a $0.22 \mu \mathrm{M}$ syringe filter and added to cells at $100 \mu \mathrm{L}$ per well. Four hours after treatment, the bioactivity of PEG-DPCAs was evaluated by immunostaining of HIF-1 $\alpha$. For immunostaining, hAECs were fixed with $4 \%$ paraformaldehyde for $10 \mathrm{~min}$ and then permeabilized with $0.3 \%$ Triton X-100 for 20 min at room temperature. To prevent nonspecific binding of antibodies, 5\% goat blocking serum was added to cells for $1 \mathrm{~h}$. Cells were then incubated with primary anti-HIF-1 $\alpha$ (1:300 dilution, ab179483, Abcam) and anti-cytokeratin-18 (CK-18; 1:200 dilution, MA119039, Thermo Scientific), as a specific marker of hAECs, at $4^{\circ} \mathrm{C}$ overnight. After multiple washes with PBS buffer, secondary antibodies, Alexa Fluor 488 (1:200, A11008, Invitrogen) and Alex Fluor 594 (1:200, A11005, Invitrogen) were treated for 45 min at room temperature. Cell nuclei were counterstained with 4',6-diamidino-2-phenylindole (DAPI) in antifade reagent (ProLong Diamond Antifade mountant with DAPI, Life Technologies). The stained cells were observed using Keyence BZX-800 microscope.

\section{References}

(1) Gurtner, G. C.; Werner, S.; Barrandon, Y.; Longaker, M. T. Wound Repair and Regeneration. Nature 2008, 453 (7193), 314-321. https://doi.org/10.1038/nature07039.

(2) Chocarro-Wrona, C.; López-Ruiz, E.; Perán, M.; Gálvez-Martín, P.; Marchal, J. A. Therapeutic Strategies for Skin Regeneration Based on Biomedical Substitutes. J. Eur. Acad. Dermatol. Venereol. 2019, 33 (3), 484-496. https://doi.org/10.1111/jdv.15391.

(3) Boateng, J. S.; Matthews, K. H.; Stevens, H. N. E.; Eccleston, G. M. Wound Healing Dressings and Drug Delivery Systems: A Review. J. Pharm. Sci. 2008, 97 (8), 2892-2923. https://doi.org/10.1002/jps.21210.

(4) Mao, A. S.; Mooney, D. J. Regenerative Medicine: Current Therapies and Future Directions. Proc. Natl. Acad. Sci. 2015, 112 (47), 14452 LP - 14459. https://doi.org/10.1073/pnas.1508520112.

(5) Huang, N. F.; Serpooshan, V.; Morris, V. B.; Sayed, N.; Pardon, G.; Abilez, O. J.; Nakayama, K. H.; Pruitt, B. L.; Wu, S. M.; Yoon, Y.; Zhang, J.; Wu, J. C. Big Bottlenecks in Cardiovascular Tissue Engineering. Commun. Biol. 2018, 1 (1), 199. https://doi.org/10.1038/s42003-018-0202-8.

(6) Cheng, J.; Amin, D.; Latona, J.; Heber-Katz, E.; Messersmith, P. B. Supramolecular Polymer Hydrogels for Drug-Induced Tissue Regeneration. ACS Nano 2019, 13 (5), 5493-5501. https://doi.org/10.1021/acsnano.9b00281.

(7) Zhang, Y.; Strehin, I.; Bedelbaeva, K.; Gourevitch, D.; Clark, L.; Leferovich, J.; Messersmith, P. B.; Heber-Katz, E. Drug-Induced Regeneration in Adult Mice. Sci. Transl. Med. 2015, 7 (290), 290ra92-290ra92. https://doi.org/10.1126/scitranslmed.3010228.

(8) Nagai, K.; Ideguchi, H.; Kajikawa, T.; Li, X.; Chavakis, T.; Cheng, J.; Messersmith, P. B.; Heber- 
Katz, E.; Hajishengallis, G. An Injectable Hydrogel-Formulated Inhibitor of Prolyl-4-Hydroxylase Promotes T Regulatory Cell Recruitment and Enhances Alveolar Bone Regeneration during Resolution of Experimental Periodontitis. FASEB J. 2020, 34 (10), 13726-13740.

https://doi.org/https://doi.org/10.1096/fj.202001248R.

(9) $\quad$ Fan, F.; He, Z.; Kong, L.-L.; Chen, Q.; Yuan, Q.; Zhang, S.; Ye, J.; Liu, H.; Sun, X.; Geng, J.; Yuan, L.; Hong, L.; Xiao, C.; Zhang, W.; Sun, X.; Li, Y.; Wang, P.; Huang, L.; Wu, X.; Ji, Z.; Wu, Q.; Xia, N.-S.; Gray, N. S.; Chen, L.; Yun, C.-H.; Deng, X.; Zhou, D. Pharmacological Targeting of Kinases MST1 and MST2 Augments Tissue Repair and Regeneration. Sci. Transl. Med. 2016, 8 (352), 352ra108. https://doi.org/10.1126/scitranslmed.aaf2304.

(10) Zhang, Y.; Desai, A.; Yang, S. Y.; Bae, K. B.; Antczak, M. I.; Fink, S. P.; Tiwari, S.; Willis, J. E.; Williams, N. S.; Dawson, D. M.; Wald, D.; Chen, W.-D.; Wang, Z.; Kasturi, L.; Larusch, G. A.; He, L.; Cominelli, F.; Di Martino, L.; Djuric, Z.; Milne, G. L.; Chance, M.; Sanabria, J.; Dealwis, C.; Mikkola, D.; Naidoo, J.; Wei, S.; Tai, H.-H.; Gerson, S. L.; Ready, J. M.; Posner, B.; Willson, J. K. V; Markowitz, S. D. TISSUE REGENERATION. Inhibition of the Prostaglandin-Degrading Enzyme 15-PGDH Potentiates Tissue Regeneration. Science 2015, 348 (6240), aaa2340-aaa2340. https://doi.org/10.1126/science.aaa2340.

(11) Zeng, Y.; Shih, Y.-R. V; Baht, G. S.; Varghese, S. In Vivo Sequestration of Innate Small Molecules to Promote Bone Healing. Adv. Mater. 2020, 32 (8), 1906022.

https://doi.org/https://doi.org/10.1002/adma.201906022.

(12) Semenza, G. L. HIF-1 and Mechanisms of Hypoxia Sensing. Curr. Opin. Cell Biol. 2001, 13 (2), 167-171. https://doi.org/10.1016/s0955-0674(00)00194-0.

(13) Hirsila, M.; Koivunen, P.; Gunzler, V.; Kivirikko, K. I.; Myllyharju, J. Characterization of the Human Prolyl 4-Hydroxylases That Modify the Hypoxia-Inducible Factor. J. Biol. Chem. 2003, 278 (33), 30772-30780.

(14) Banerji, B.; Conejo-Garcia, A.; McNeill, L. A.; McDonough, M. A.; Buck, M. R. G.; Hewitson, K. S.; Oldham, N. J.; Schofield, C. J. The Inhibition of Factor Inhibiting Hypoxia-Inducible Factor (FIH) by $\beta$-Oxocarboxylic Acids. Chem. Commun. 2005, No. 43, 5438-5440. https://doi.org/10.1039/B510707E.

(15) Franklin, T. J.; Morris, W. P.; Edwards, P. N.; Large, M. S.; Stephenson, R. Inhibition of Prolyl 4Hydroxylase in Vitro and in Vivo by Members of a Novel Series of Phenanthrolinones. Biochem. J. 2001, 353 (Pt 2), 333-338. https://doi.org/10.1042/0264-6021:3530333.

(16) J., M. N.; J., V. H.; A., M. K.; Annie, G.; L., P. Q.; M., S. M.; Cody, R.-I.; W., K. A.; L., K. D.; Michael, L. Acute Multidrug Delivery via a Wearable Bioreactor Facilitates Long-Term Limb Regeneration and Functional Recovery in Adult Xenopus Laevis. Sci. Adv. 2022, 8 (4), eabj2164. https://doi.org/10.1126/sciadv.abj2164.

(17) Lertora, J. J. L.; Vanevski, K. M. Introduction to Pharmacokinetics and Pharmacodynamics. In Small Molecule Therapy for Genetic Disease; Thoene, J. G., Ed.; Cambridge University Press: Cambridge, 2010; pp 35-54. https://doi.org/DOI: 10.1017/CBO9780511777905.004.

(18) Champion, J. A.; Mitragotri, S. Role of Target Geometry in Phagocytosis. Proc. Natl. Acad. Sci. 2006, 103 (13), 4930-4934. https://doi.org/10.1073/PNAS.0600997103.

(19) Almeida, J. P. M.; Chen, A. L.; Foster, A.; Drezek, R. In Vivo Biodistribution of Nanoparticles. Nanomedicine 2011, 6 (5), 815-835. https://doi.org/10.2217/nnm.11.79.

(20) Wang, H.; Xie, H.; Wang, J.; Wu, J.; Ma, X.; Li, L.; Wei, X.; Ling, Q.; Song, P.; Zhou, L.; Xu, X.; Zheng, S. Self-Assembling Prodrugs by Precise Programming of Molecular Structures That Contribute Distinct Stability, Pharmacokinetics, and Antitumor Efficacy. Adv. Funct. Mater. 2015, 25 (31), 4956-4965. https://doi.org/https://doi.org/10.1002/adfm.201501953.

(21) Rautio, J.; Meanwell, N. A.; Di, L.; Hageman, M. J. The Expanding Role of Prodrugs in 
Contemporary Drug Design and Development. Nat. Rev. Drug Discov. 2018, 17 (8), 559-587. https://doi.org/10.1038/nrd.2018.46.

(22) Cheetham, A. G.; Chakroun, R. W.; Ma, W.; Cui, H. Self-Assembling Prodrugs. Chem. Soc. Rev. 2017, 46 (21), 6638-6663. https://doi.org/10.1039/C7CS00521K.

(23) Wang, H.; Lu, Z.; Wang, L.; Guo, T.; Wu, J.; Wan, J.; Zhou, L.; Li, H.; Li, Z.; Jiang, D.; Song, P.; Xie, H.; Zhou, L.; Xu, X.; Zheng, S. New Generation Nanomedicines Constructed from SelfAssembling Small-Molecule Prodrugs Alleviate Cancer Drug Toxicity. Cancer Res. 2017, 77 (24), 6963. https://doi.org/10.1158/0008-5472.CAN-17-0984.

(24) Su, H.; Wang, F.; Ran, W.; Zhang, W.; Dai, W.; Wang, H.; Anderson, C. F.; Wang, Z.; Zheng, C.; Zhang, P.; Li, Y.; Cui, H. The Role of Critical Micellization Concentration in Efficacy and Toxicity of Supramolecular Polymers. Proc. Natl. Acad. Sci. U. S. A. 2020, 117 (9), 4518-4526. https://doi.org/10.1073/pnas.1913655117.

(25) Croy, S. R.; Kwon, G. S. Polymeric Micelles for Drug Delivery. Curr. Pharm. Des. 2006, 12 (36), 4669-4684. https://doi.org/http://dx.doi.org/10.2174/138161206779026245.

(26) Ahmad, Z.; Shah, A.; Siddiq, M.; Kraatz, H.-B. Polymeric Micelles as Drug Delivery Vehicles. RSC Adv. 2014, 4 (33), 17028-17038. https://doi.org/10.1039/C3RA47370H.

(27) Aiertza, M. K.; Odriozola, I.; Cabañero, G.; Grande, H.-J.; Loinaz, I. Single-Chain Polymer Nanoparticles. Cell. Mol. Life Sci. 2012, 69 (3), 337-346. https://doi.org/10.1007/s00018-0110852-x.

(28) Lawrence, M. J. Surfactant Systems: Their Use in Drug Delivery. Chem. Soc. Rev. 1994, 23 (6), 417-424. https://doi.org/10.1039/CS9942300417.

(29) Lin, R.; Cheetham, A. G.; Zhang, P.; Lin, Y.; Cui, H. Supramolecular Filaments Containing a Fixed 41\% Paclitaxel Loading. Chem. Commun. 2013, 49 (43), 4968-4970. https://doi.org/10.1039/C3CC41896K.

(30) Goodwin, A. P.; Mynar, J. L.; Ma, Y.; Fleming, G. R.; Fréchet, J. M. J. Synthetic Micelle Sensitive to IR Light via a Two-Photon Process. J. Am. Chem. Soc. 2005, 127 (28), 9952-9953. https://doi.org/10.1021/ja0523035.

(31) Poelma, S. O.; Oh, S. S.; Helmy, S.; Knight, A. S.; Burnett, G. L.; Soh, H. T.; Hawker, C. J.; Read de Alaniz, J. Controlled Drug Release to Cancer Cells from Modular One-Photon Visible LightResponsive Micellar System. Chem. Commun. 2016, 52 (69), 10525-10528. https://doi.org/10.1039/C6CC04127B.

(32) Nagy, M.; Zsuga, M.; Rácz, D.; Kéki, S. Synthesis of Phenanthroline-Terminated Polymers and Their Fe(II)-Complexes. J. Polym. Sci. Part A Polym. Chem. 2010, 48 (12), 2709-2715. https://doi.org/https://doi.org/10.1002/pola.24054.

(33) Kuki, Á.; Nagy, M.; Nagy, L.; Zsuga, M.; Kéki, S. Ligand Size Distribution of PhenanthrolineFunctionalized Polyethylene Glycol-Iron(II) Complexes Determined by Electrospray lonization Mass Spectrometry and Computer Simulation. J. Am. Soc. Mass Spectrom. 2010, 21 (9), 15611564. https://doi.org/https://doi.org/10.1016/j.jasms.2010.04.019.

(34) Oesterhelt, F.; Rief, M.; Gaub, H. E. Single Molecule Force Spectroscopy by AFM Indicates Helical Structure of Poly(Ethylene-Glycol) in Water. New J. Phys. 1999, 1, 6. https://doi.org/10.1088/1367-2630/1/1/006.

(35) Geng, Y.; Dalhaimer, P.; Cai, S.; Tsai, R.; Tewari, M.; Minko, T.; Discher, D. E. Shape Effects of Filaments versus Spherical Particles in Flow and Drug Delivery. Nat. Nanotechnol. 2007, 2 (4), 249-255. https://doi.org/10.1038/nnano.2007.70.

(36) Hoshyar, N.; Gray, S.; Han, H.; Bao, G. The Effect of Nanoparticle Size on in Vivo 
Pharmacokinetics and Cellular Interaction. Nanomedicine 2016, 11 (6), 673-692.

(37) Israelachvili, J. N. Intermolecular and Surface Forces, 3rd ed.; Academic Press, Elsevier, 2011.

(38) Allinger, N. L. Conformational Analysis. 130. MM2. A Hydrocarbon Force Field Utilizing V1 and V2 Torsional Terms. J. Am. Chem. Soc. 2002, 99 (25), 8127-8134. https://doi.org/10.1021/JA00467A001.

(39) Nguyen, T. D.; Phillips, C. L.; Anderson, J. A.; Glotzer, S. C. Rigid Body Constraints Realized in Massively-Parallel Molecular Dynamics on Graphics Processing Units. Comput. Phys. Commun. 2011, 182 (11), 2307-2313. https://doi.org/10.1016/J.CPC.2011.06.005.

(40) Arkın, H.; Janke, W. Citation. J. Chem. Phys. 2013, 138, 54904. https://doi.org/10.1063/1.4788616.

(41) Beck, V.; Lewi, P.; Gucciardo, L.; Devlieger, R. Preterm Prelabor Rupture of Membranes and Fetal Survival after Minimally Invasive Fetal Surgery: A Systematic Review of the Literature. Fetal Diagn. Ther. 2012, 31 (1), 1-9. https://doi.org/10.1159/000331165.

(42) Fong, G.-H.; Takeda, K. Role and Regulation of Prolyl Hydroxylase Domain Proteins. Cell Death Differ. 2008, 15 (4), 635-641. https://doi.org/10.1038/cdd.2008.10.

(43) Kremer, K.; Grest, G. S. Dynamics of Entangled Linear Polymer Melts: A Molecular-dynamics Simulation. J. Chem. Phys. 1998, 92 (8), 5057. https://doi.org/10.1063/1.458541.

(44) Weeks, J. D.; Chandler, D.; Andersen, H. C. Role of Repulsive Forces in Determining the Equilibrium Structure of Simple Liquids. J. Chem. Phys. 2003, 54 (12), 5237. https://doi.org/10.1063/1.1674820.

(45) Anderson, J. A.; Glaser, J.; Glotzer, S. C. HOOMD-Blue: A Python Package for High-Performance Molecular Dynamics and Hard Particle Monte Carlo Simulations. Comput. Mater. Sci. 2020, 173, 109363. https://doi.org/10.1016/J.COMMATSCI.2019.109363.

(46) Stukowski, A. Visualization and Analysis of Atomistic Simulation Data with OVITO-the Open Visualization Tool. Model. Simul. Mater. Sci. Eng. 2009, 18 (1), 015012.

https://doi.org/10.1088/0965-0393/18/1/015012. 